\title{
Analysis of Differential Orthogonal Space-Time Block Codes over Semi-Identical MIMO Fading Channels
}

\author{
Meixia Tao and Pooi Yuen Kam \\ Department of Electrical and Computer Engineering \\ National University of Singapore \\ Singapore 117576 \\ Email: $\{$ mxtao, elekampy $\} @$ nus.edu.sg
}

\begin{abstract}
We study the performance of differential orthogonal space-time block codes over independent and semi-identically distributed Rayleigh fading channels. In this semi-identically distributed fading model, the channel gains from different transmit antennas to a common receive antenna are identically distributed, but the gains associated with different receive antennas are non-identically distributed. Arbitrary fluctuation rates of the fading processes from one transmission block to another are considered. We first derive the optimal symbolby-symbol differential detector, and show that the conventional differential detector is suboptimal. We then derive expressions of exact bit error probabilities (BEP) for both the optimal and suboptimal detectors. The results are applicable for any number of receive antennas and any number of transmit antennas for which orthogonal space-time block codes exist. For two transmit antennas, explicit and closed-form BEP expressions are obtained. A Chernoff bound on the BEP of optimal detection for any number of transmit antennas is also derived.
\end{abstract}

\section{INTRODUCTION}

Performance analysis for diversity combining techniques commonly assumes identically distributed diversity branches, due to its analytical tractability. In practice, however, this assumption may not hold. For instance, the environmental scattering on the propagation paths observed by different branches may be different. This may incur shadowing effects and, consequently, unequal path loss on different branches. In multi-input multi-output (MIMO) systems, this is especially common when the antenna spacing is relatively large (compared with the carrier wavelength) to ensure low correlation. The performance of diversity reception over non-identically distributed branches in single-input multi-output (SIMO) systems has been studied in, for example, [1], [2]. The goal of this paper is to investigate the effects of non-identical distribution on the performance of joint diversity transmission and reception in MIMO systems.

Of the existing transmit diversity techniques, orthogonal space-time block codes (OSTBC) [3]-[5] are most popular because of their elegant code structure and low decoding complexity. The performance of OSTBC is known to be identical to that of maximum ratio combing in SIMO channels (apart from being scaled down by the number of transmit antennas and coding rate) when channel state information
(CSI) is available at the receiver. When CSI is unavailable, OSTBC can be used in a differential encoding manner and still possess low decoding complexity [6]-[8]. Existing work on performance analysis of differential OSTBC can be found in [9]-[11], and they all assume identical channel distributions.

We concentrate on the analysis of differential OSTBC over independent, semi-identically distributed (i.s.i.d) and blockwise time-varying Rayleigh fading channels. The i.s.i.d fading model refers to the situations where the channel gains from different transmit antennas to a common receive antenna are identically distributed, but the gains associated with different receive antennas are non-identically distributed. Such situations would most likely occur in the uplink of a cellular system, where the antennas on the base station are mounted several wavelengths apart from one another, whereas the antennas on a mobile handset are inside a small antenna panel. In the general independent and non-identically distributed (i.n.i.d) fading channels, a novel decomposition for the longterm channel parameters in conjunction with proper power allocation can be applied. By doing so, the i.n.i.d case can be equivalent to the i.s.i.d one and, hence, the results derived for i.s.i.d channels here can be directly applied. In the blockwise time-varying fading model, the channel coefficients are assumed to keep constant over one space-time block code transmission but fluctuate from one block to another at an arbitrary fluctuating rate.

The rest of this paper is organized as follows. In Section II we introduce the non-identical and semi-identical MIMO channel models. In Section III we present the optimal and suboptimal detectors of differential OSTBC. The derivations of exact BEP and their bounds are provided in Section IV. Numerical results and discussions are presented in Section V. Finally we conclude this paper in Section VI.

\section{Channel Model}

We consider a wireless communication link equipped with $M$ transmit antennas and $N$ receive antennas. The channel is assumed to be frequency nonselective Rayleigh fading and block-wise time-varying, with each block consisting of $L$ symbol intervals. It is also assumed that the channel coefficients 
between any transmit-receive antenna pairs are independent. Let $\mathbf{H}[k]$ denote the $M \times N$ channel gain matrix during the $k$-th transmission block. The $(m, n)$-th entry $h_{m, n}[k]$ is the fading process of the channel between the $m$-th transmit antenna and the $n$-th receive antenna. Each $h_{m, n}[k]$ is modeled as samples of a complex Gaussian random variable with zero mean and variance $2 R_{m, n}[0]$. In general, $R_{m, n}[0]$ is not necessarily the same for all $1 \leq m \leq M$ and $1 \leq n \leq N$. The autocorrelation function of each sequence $\left\{h_{m, n}[k]\right\}_{k}$ under the block-wise time-varying fading assumption is denoted as $2 R_{m, n}[l]=E\left[h_{m, n}[k] h_{m, n}^{*}[k-l]\right]$. The fading correlation coefficient across two adjacent blocks, called block correlation coefficient, is given by $\rho_{m, n}=R_{m, n}[1] / R_{m, n}[0]$, and is a measure of the fluctuation rate of the channel fading process. In practice, the Doppler frequency shift experienced by the communication links due to relative movement of the sender and receiver would likely be the same for all transmit-receive antenna pairs. As a result, $\rho_{m, n}$ will likely be the same for all $m$ 's and $n$ 's. Nevertheless, we assume that they are not the same, unless otherwise stated, for the sake of generality.

In this work we consider only the semi-identical channels with $R_{m, n}[l]=R_{n}[l]$, for all $m$. That is, the path gains from different transmit antennas to a common receive antenna are identically distributed. This channel model is general in the sense that the non-identical channels can also be treated as the semi-identical ones under certain conditions. Specifically, we define an $M N \times M N$ diagonal matrix $\boldsymbol{\Delta}$ whose $[(n-$ 1) $M+m]$-th diagonal entry is $2 R_{m, n}[0]$, for $1 \leq m \leq M$ and $1 \leq n \leq N$. If $\rho_{m, n}=\rho$, for all $m$ and $n$, and $\boldsymbol{\Delta}$ can be decoupled as $\boldsymbol{\Delta}=\boldsymbol{\Delta}_{T} \otimes \boldsymbol{\Delta}_{R}$, where $\otimes$ stands for the Kronecker product, and $\boldsymbol{\Delta}_{T}$ and $\boldsymbol{\Delta}_{R}$ are $M \times M$ and $N \times N$ diagonal matrices, respectively, then the statistical properties of $\mathbf{H}[k]$ are identical to those of the matrix $\boldsymbol{\Delta}_{T}^{1 / 2} \tilde{\mathbf{H}}[k] \boldsymbol{\Delta}_{R}^{1 / 2}$. We denote the statistical equivalence as

$$
\mathbf{H}[k] \sim \boldsymbol{\Delta}_{T}^{1 / 2} \tilde{\mathbf{H}}[k] \boldsymbol{\Delta}_{R}^{1 / 2} .
$$

Here, $\boldsymbol{\Delta}_{T}^{1 / 2}\left(\boldsymbol{\Delta}_{T}^{1 / 2}\right)^{H}=\boldsymbol{\Delta}_{T}, \boldsymbol{\Delta}_{R}^{1 / 2}\left(\boldsymbol{\Delta}_{R}^{1 / 2}\right)^{H}=\boldsymbol{\Delta}_{R}$, and the $M \times N$ matrix $\tilde{\mathbf{H}}[k]$ contains independent and identically distributed (i.i.d) entries, each of which is modeled as samples of a complex zero-mean Gaussian random process having autocorrelation function $2 R[l]$, with $2 R[0]=1$ and $2 R[1]=\rho$. Therefore, a power allocation matrix $\mathbf{P}=\left(\boldsymbol{\Delta}_{T}^{1 / 2}\right)^{-1}$ can be applied at the transmitter such that the effective channel gain matrix $\mathbf{P H}[k]$ becomes semi-identical. All the results obtained in this paper can then be used directly in the general i.n.i.d channels

The baseband channel input-output relationship can be modeled as

$$
\mathbf{Y}[k]=\sqrt{E_{s}} \mathbf{X}[k] \mathbf{H}[k]+\mathbf{W}[k],
$$

where $\mathbf{X}[k], \mathbf{Y}[k]$, and $\mathbf{W}[k]$ are the $L \times M$ transmitted signal matrix, $L \times N$ received signal matrix and $L \times N$ noise matrix, respectively, during the $k$-th block. $\mathbf{X}[k]$ satisfies the average power constraint: $E\left[\operatorname{tr}\left(\mathbf{X}[k] \mathbf{X}^{H}[k]\right)\right]=L$. The entries of $\mathbf{W}[k]$ are i.i.d, each with mean zero and variance $N_{0} . E_{s}$ is the average total energy transmitted on all antennas.

\section{Optimal And Suboptimal DifFerential DETECTION OF OSTBC}

In this section we first review differential transmission of OSTBC, and then propose the optimal symbol-by-symbol differential detector over the i.s.i.d MIMO channel. We show that the conventional differential detector described in [8] is suboptimal, and that the optimal detector should involve weighting the output from each receive antenna according to its channel statistical information.

In the differential transmission of a square OSTBC codeword, the block length equals the number of transmit antennas, i.e., $L=M$. Let $\left\{s_{p}\right\}_{p=1}^{P}$ denote a set of $P$ information symbols to be transmitted in the $k$-th block. They are from MPSK (M-ary phase shift keying) constellations with $s_{p}=$ $e^{j \theta_{p}}$ and encoded in an $M \times M$ OSTBC codeword as

$$
\mathbf{D}[k]=\frac{1}{\sqrt{P}} \sum_{p=1}^{P}\left(\boldsymbol{\Phi}_{p} \cos \theta_{p}+j \boldsymbol{\Psi}_{p} \sin \theta_{p}\right),
$$

where the $M \times M$ encoding matrices $\boldsymbol{\Phi}_{p}$ and $\boldsymbol{\Psi}_{p}$ are linked to the theory of amicable orthogonal designs [12]-[14]. The data matrix $\mathbf{D}[k]$ is differentially encoded in the transmitted signal matrix $\mathbf{X}[k]$ as $\mathbf{X}[k]=\mathbf{D}[k] \mathbf{X}[k-1]$. Since $\mathbf{D}[k]$ is unitary, $\mathbf{X}[k]$ is also unitary.

We consider the maximum likelihood (ML) detection of $\mathbf{D}[k]$ based on two consecutive received matrices $\mathbf{Y}[k]$ and $\mathbf{Y}[k-1]$. Define $\mathbf{Y}=\left[\mathbf{Y}^{T}[k-1], \quad \mathbf{Y}^{T}[k]\right]^{T}$ and $\mathbf{y}_{n}=$ $\left[\mathbf{y}_{n}^{T}[k-1], \mathbf{y}_{n}^{T}[k]\right]^{T}$, where $\mathbf{y}_{n}[k]$ is the received signal vector on the $n$-th antenna during the $k$-th block for $n=1, \ldots, N$. Conditioned on $\mathbf{D}[k]$ and $\mathbf{X}[k-1]$, the $2 M \times 1$ vectors $\mathbf{y}_{n}$ 's can be shown to be independent and each has a complex Gaussian distribution with mean zero and covariance matrix

$$
\boldsymbol{\Lambda}_{n}=E_{s}\left[\begin{array}{cc}
2 R_{n}[0] \mathbf{I}_{M} & 2 R_{n}[1] \mathbf{D}^{H} \\
2 R_{n}[1] \mathbf{D} & 2 R_{n}[0] \mathbf{I}_{M}
\end{array}\right]+N_{0} \mathbf{I}_{2 M}
$$

In (2), the block index in $\mathbf{D}[k]$ is omitted for notational brevity. Using the matrix formulas [15]

$$
\begin{aligned}
(\mathbf{A}+\mathbf{B C D})^{-1} & =\mathbf{A}^{-1}-\mathbf{A}^{-1} \mathbf{B}\left(\mathbf{C}^{-1}+\mathbf{D A} \mathbf{A}^{-1} \mathbf{B}\right)^{-1} \mathbf{D A}^{-1} \\
\operatorname{det}\left[\begin{array}{ll}
\mathbf{A} & \mathbf{B} \\
\mathbf{C} & \mathbf{D}
\end{array}\right] & =\operatorname{det}\left(\mathbf{A}-\mathbf{B D}^{-1} \mathbf{C}\right) \operatorname{det} \mathbf{D}
\end{aligned}
$$

we obtain

$$
\begin{aligned}
\left(\boldsymbol{\Lambda}_{n}\right)^{-1}= & \frac{1}{N_{0}}\left(\mathbf{I}-\frac{\gamma_{n}}{\left(1+\gamma_{n}\right)^{2}-\left(\rho_{n} \gamma_{n}\right)^{2}}\right. \\
& {\left.\left[\begin{array}{cc}
{\left[1+\gamma_{n}\left(1-\rho_{n}^{2}\right)\right] \mathbf{I}} & \rho_{n} \mathbf{D}^{H} \\
\rho_{n} \mathbf{D} & {\left[1+\gamma_{n}\left(1-\rho_{n}^{2}\right)\right] \mathbf{I}}
\end{array}\right]\right)(4) }
\end{aligned}
$$

and

$$
\operatorname{det}\left(\boldsymbol{\Lambda}_{n}\right)=N_{0}^{2 M}\left[\left(1+\gamma_{n}\right)^{2}-\left(\rho_{n} \gamma_{n}\right)^{2}\right]^{M} .
$$

In both (4) and (5), $\gamma_{n} \triangleq 2 E_{s} R_{n}[0] / N_{0}$ is the average received signal-to-noise ratio (SNR) on the $n$-th antenna. Taking the natural logarithm of the conditional probability density function of $\mathbf{Y}$ and ignoring the terms that are independent of $\mathbf{D}$, 
we obtain the ML decision metric of $\mathbf{D}$ as

$$
\hat{\mathbf{D}}=\arg \max _{\mathbf{D}} \Re\left\{\sum_{n=1}^{N} w_{n} \mathbf{y}_{n}^{H}[k-1] \mathbf{D}^{H} \mathbf{y}_{n}[k]\right\}
$$

where $\Re(x)$ stands for the real part of $x$, and $w_{n}$ is given by

$$
w_{n}=\frac{\rho_{n} \gamma_{n}}{\left(1+\gamma_{n}\right)^{2}-\left(\rho_{n} \gamma_{n}\right)^{2}} .
$$

Here, we assume that the channel second-order statistics, $\rho_{n}$ 's and $\gamma_{n}$ 's, vary much more slowly than the instantaneous channel coefficients and hence can be estimated at the receiver. Now, using the inherent linear structure of the data matrix by design in (1), the above matrix-by-matrix detector reduces to a symbol-by-symbol detector given by

$$
\hat{s}_{p}=\arg \max _{s_{p}} \Re\left\{\sum_{n=1}^{N} w_{n} z_{n, p} s_{p}^{*}\right\}, p=1, \ldots, P
$$

where

$z_{n, p}=\Re\left\{\mathbf{y}_{n}^{H}[k-1] \boldsymbol{\Phi}_{p}^{H} \mathbf{y}_{n}[k]\right\}-j \Re\left\{j \mathbf{y}_{n}^{H}[k-1] \boldsymbol{\Psi}_{p}^{H} \mathbf{y}_{n}[k]\right\}$ is the contribution to the decision variable from the $n$-th receive antenna. The contributions from different receive antennas are weighted by the $w_{n}$ 's. Note that the optimal weights in (6) coincide with the result in [1] for SIMO channels. A detailed discussion on the weights will be provided in Section V.

When the channel second-order statistics are unknown at the receiver, one would let the contributions from different receive antennas be weighted equally in the detector. Thus by letting $w_{n}=1$ for all $n$, (7) reduces to

$$
\hat{s}_{p}=\arg \max _{s_{p}} \Re\left\{\sum_{n=1}^{N} z_{n, p} s_{p}^{*}\right\}, p=1, \ldots, P
$$

which agrees with the decision rule in [8], [10] for identically distributed channels. Hence, the traditional differential detector is suboptimal in the i.s.i.d case.

\section{EXACT BEP AND UPPER BOUNDS}

In this section, we derive exact BEP expressions for both the optimal and suboptimal detectors. The results are for any number of receive antennas and any number of transmit antennas for which OSTBCs exist. In the case with two transmit antennas, explicit and closed-form expressions are obtained. An insightful upper bound for an arbitrary number of transmit antennas is also derived.

The analysis of BEP for detecting symbol $s_{p}$ involves the computation of the following probability [16]

$$
F\left(\alpha \mid \theta_{p}\right)=P\left(Z_{p}(\alpha)<0 \mid s_{p}=e^{j \theta_{p}}\right)
$$

where

$$
Z_{p}(\alpha)=\Re\left(\sum_{n=1}^{N} w_{n} z_{n, p} e^{j \alpha}\right)
$$

is the decision phasor for $s_{p}$, and $\alpha$ is some angle. It will be shown later that the above probability is the same for all $p$ 's, and thus the BEP of $s_{p}$ corresponds also to the overall BEP.
The decision phasor in (10) can be written as the Hermitian quadratic form of Gaussian vectors:

$$
Z_{p}(\alpha)=\frac{1}{2} \sum_{n=1}^{N} w_{n} \mathbf{y}_{n}^{H} \boldsymbol{\Omega}_{p} \mathbf{y}_{n}
$$

where $\boldsymbol{\Omega}_{p}$ is given by

$$
\boldsymbol{\Omega}_{p}=\left[\begin{array}{cc}
\mathbf{0} & \cos \alpha \boldsymbol{\Phi}_{p}^{H}+j \sin \alpha \boldsymbol{\Psi}_{p}^{H} \\
\cos \alpha \boldsymbol{\Phi}_{p}-j \sin \alpha \boldsymbol{\Psi}_{p} & \mathbf{0}
\end{array}\right]
$$

Applying the cumulative distribution function of a Hermitian quadratic form [17], we have

$$
F\left(\alpha \mid \theta_{p}\right)=-\sum_{\lambda_{n, i}<0} \operatorname{Res}\left[\frac{\phi(s)}{s}, s=\frac{-2}{w_{n} \lambda_{n, i}}\right]
$$

where $\operatorname{Res}\left[f(x), x_{i}\right]$ denotes the residue of $f(x)$ at $x_{i}$, $\left\{\lambda_{n, i}\right\}_{i=1}^{K}$ is the set of nonzero distinct eigenvalues of the matrix $\boldsymbol{\Lambda}_{n} \boldsymbol{\Omega}_{p}$ with multiplicities $\left\{u_{n, i}\right\}_{i=1}^{K}$, and

$$
\phi(s) \triangleq \frac{1}{\prod_{n=1}^{N} \prod_{i=1}^{K}\left(1+s \frac{w_{n} \lambda_{n, i}}{2}\right)^{u_{n, i}}} .
$$

We investigate below the eigenvalues of $\mathbf{A}_{n}=\boldsymbol{\Lambda}_{n} \boldsymbol{\Omega}_{p}$, for $n=1, \ldots, N$.

Let $\mathbf{A}_{n}$ undergo a similitude transformation producing

$$
\tilde{\mathbf{A}}_{n}=\boldsymbol{\Theta} \mathbf{A}_{n} \boldsymbol{\Theta}^{H}
$$

where $\boldsymbol{\Theta}$ is an $2 M \times 2 M$ unitary matrix. Then the eigenvalues of $\mathbf{A}_{n}$ are the same as those of $\tilde{\mathbf{A}}_{n}$. Here we choose

$$
\Theta=\left[\begin{array}{ll}
\mathbf{I} & \mathbf{0} \\
\mathbf{0} & \mathbf{D}
\end{array}\right] \text {. }
$$

Substituting (12), (2) and (15) into (14) yields

$$
\tilde{\mathbf{A}}_{n}=N_{0}\left[\begin{array}{cc}
\rho_{n} \gamma_{n} \mathbf{D}^{H} \mathbf{Q}^{H} & \left(1+\gamma_{n}\right) \mathbf{Q D} \\
\left(1+\gamma_{n}\right) \mathbf{D}^{H} \mathbf{Q}^{H} & \rho_{n} \gamma_{n} \mathbf{Q D}
\end{array}\right],
$$

in which

$$
\mathbf{Q}=\cos \alpha \boldsymbol{\Phi}_{p}^{H}+j \sin \alpha \boldsymbol{\Psi}_{p}^{H} .
$$

Now we are ready to evaluate the eigenvalues of $\tilde{\mathbf{A}}_{n}$ directly. The eigenvalues of $\tilde{\mathbf{A}}_{n}$ are the solutions of

$$
\operatorname{det}\left(\tilde{\mathbf{A}}_{n}-\lambda \mathbf{I}_{2 M}\right)=0 .
$$

By using the matrix formula (3) and

$$
\operatorname{det}(\mathbf{A B})=\operatorname{det}(\mathbf{A}) \operatorname{det}(\mathbf{B})
$$

(16) becomes

$$
\begin{aligned}
\left(\frac{\lambda}{N_{0}}\right)^{2}-\left(\frac{\lambda}{N_{0}}\right) & {\left[\frac{2}{\sqrt{P}} \rho_{n} \gamma_{n} \cos \left(\alpha+\theta_{p}\right)\right] } \\
& -\left[\left(1+\gamma_{n}\right)^{2}-\left(\rho_{n} \gamma_{n}\right)^{2}\right]=0 .
\end{aligned}
$$

In obtaining (17), the following two equations are used:

$$
\begin{gathered}
\mathbf{Q D}+\mathbf{D}^{H} \mathbf{Q}^{H}=\frac{2}{\sqrt{P}} \cos \left(\alpha+\theta_{p}\right) \mathbf{I} \\
\mathbf{D}^{H} \mathbf{Q}^{H}\left(\rho_{n} \gamma_{n} \mathbf{Q D}-\frac{\lambda}{N_{0}} \mathbf{I}\right)=\left(\rho_{n} \gamma_{n} \mathbf{Q D}-\frac{\lambda}{N_{0}} \mathbf{I}\right) \mathbf{D}^{H} \mathbf{Q}^{H} .
\end{gathered}
$$


Finally, solving the quadratic equation (17) produces the two distinct eigenvalues of $\tilde{\mathbf{A}}_{n}$ as:

$$
\begin{aligned}
& \lambda_{n, i}=N_{0}\left[\frac{\rho_{n} \gamma_{n} \cos \left(\alpha+\theta_{p}\right)}{\sqrt{P}}\right. \\
& \left. \pm \sqrt{\frac{1}{P}\left(\rho_{n} \gamma_{n}\right)^{2} \cos ^{2}\left(\alpha+\theta_{p}\right)+\left(1+\gamma_{n}\right)^{2}-\left(\rho_{n} \gamma_{n}\right)^{2}}\right]
\end{aligned}
$$

for $i=1,2$. Since the $2 M \times 2 M$ matrix $\tilde{\mathbf{A}}_{n}$ has full rank, the multiplicity of each of the eigenvalues is $u_{n, i}=M$. We choose $\lambda_{n, 1}<0$ and $\lambda_{n, 2}>0$. The evaluation of the probability $F\left(\alpha \mid \theta_{p}\right)$ can thus be accomplished by computing the residues in (13) at $\lambda_{n, 1}, \forall n$. For a general formula of computing these residues, readers are referred to [18, D3].

Now we can compute the BEP. For BPSK and QPSK (with Gray mapping) constellations, the exact BEP is given using the half-plane decision region approach [16], respectively, by

$$
P_{b, \mathrm{BPSK}}=F\left(\alpha=0 \mid \theta_{p}=0\right)
$$

and

$$
P_{b, \mathrm{QPSK}}=F\left(\alpha=-\pi / 4 \mid \theta_{p}=0\right) .
$$

In what follows we consider the special case where $M=$ $P=2$, which is the Alamouti's space-time block code [3]. According to the residue equation of a function $f(x)$ with a pole $a$ of multiplicity $m$

$$
\operatorname{Res}\{f(x), a\}=\frac{1}{(m-1) !} \lim _{x \rightarrow a} \frac{d^{m-1}}{d x^{m-1}}\left[(x-a)^{m} f(x)\right],
$$

we can have

$$
F\left(\alpha \mid \theta_{p}\right)=\sum_{n=1}^{N} a_{n} A_{n, 1} A_{n, 2}\left(1+b_{n}+B_{n, 1}+B_{n, 2}\right)
$$

where

$$
\begin{gathered}
a_{n}=\left(1-\frac{\lambda_{n, 2}}{\lambda_{n, 1}}\right)^{-2}, \quad A_{n, i}=\prod_{\substack{k=1 \\
k \neq n}}^{N}\left(1-\frac{w_{k} \lambda_{k, i}}{w_{n} \lambda_{n, 1}}\right)^{-2}, \\
b_{n}=2\left(1-\frac{\lambda_{n, 1}}{\lambda_{n, 2}}\right)^{-1}, \quad B_{n, i}=2 \sum_{\substack{k=1 \\
k \neq n}}^{N}\left(1-\frac{w_{n} \lambda_{n, 1}}{w_{k} \lambda_{k, i}}\right)^{-1} .
\end{gathered}
$$

Eqn. (21) is a closed-form expression and depends explicitly on the average receive SNR on each antenna $\gamma_{n}$ and the block correlation coefficients $\rho_{n}$. The exact BEP of the optimal detector for BPSK and QPSK with $M=2$ can be obtained by substituting (21) into (19) and (20), respectively.

For the suboptimal detector (8), the weights satisfy $w_{n}=1$ for all $n$. Thus, the BEP expressions of the suboptimal detector are identical to those of the optimal detector by letting $w_{n}=1$ in (13) for general $M$, or in (21) for $M=2$.

Based on the eigenvalues obtained above, a Chernoff bound for $F\left(\alpha \mid \theta_{p}\right)$ can also be obtained. Applying the well-known Cherenoff bound inequality [19] to (9) and using the characteristic function for a Hermitian quadratic form of Gaussian vectors, we have

$$
F\left(\alpha \mid \theta_{p}\right) \leq E\left[e^{s Z_{p}(\alpha)}\right]=\prod_{n=1}^{N}\left[\frac{1}{c_{n}(s)}\right]^{M}
$$

in which

$$
c_{n}(s)=\left(1+s \frac{w_{n} \lambda_{n, 1}}{2}\right)\left(1+s \frac{w_{n} \lambda_{n, 2}}{2}\right),
$$

$s \geq 0$ is the parameter to be optimized, and $\lambda_{n, i}$ 's, for $i=$ 1,2 and $n=1, \ldots, N$ are given in (18). The tightest bound on $F\left(\alpha \mid \theta_{p}\right)$ can be obtained by selecting the value of $s$ that minimizes the right-hand side of the inequality (22). This is equivalent to selecting $s$ to maximize $\prod_{n=1}^{N} c_{n}(s)$. The value of $s$ that maximizes the $n$-th factor is determined by solving the equation $d c_{n}(s) / d s=0$ which gives the result

$$
s=-\frac{\lambda_{n, 1}+\lambda_{n, 2}}{w_{n} \lambda_{n, 1} \lambda_{n, 2}} .
$$

Substituting (6) and (18) into (23), we obtain

$$
s=\frac{2 \cos \left(\alpha+\theta_{p}\right)}{\sqrt{P} N_{0}} .
$$

This value is independent of the index $n$ and, hence, also maximizes $\prod_{n=1}^{N} c_{n}(s)$. Therefore, substituting (24) into (22), we have

$$
F\left(\alpha \mid \theta_{p}\right) \leq \prod_{n=1}^{N}\left\{1+\frac{\left(\rho_{n} \gamma_{n}\right)^{2} \cos ^{2}\left(\alpha+\theta_{p}\right)}{P\left[\left(1+\gamma_{n}\right)^{2}-\left(\rho_{n} \gamma_{n}\right)^{2}\right]}\right\}^{-M}
$$

The Chernoff bound in (25) can be tightened further by a factor of $1 / 2$. Thus, the Chernoff bounds on the BEP using BPSK and QPSK modulation can be found by letting $\alpha=0$ and $\alpha=-\pi / 4$ in (25), respectively, with $\theta_{p}=0$.

We now study the asymptotic behavior of the Chernoff bound for high SNRs. We first consider a static channel with $\rho_{n}=1$ for all $n$. By letting $\gamma_{n} \gg 1$, the inequality (25) can be approximated by

$$
F\left(\alpha \mid \theta_{p}\right) \leq\left[\frac{\cos ^{2}\left(\alpha+\theta_{p}\right)}{2 P} \gamma_{\mathrm{gm}}\right]^{-M N},
$$

where $\gamma_{\mathrm{gm}}$ is the geometric mean of $\left\{\gamma_{n}\right\}$, given by $\gamma_{\mathrm{gm}}=$ $\left(\prod_{n=1}^{N} \gamma_{n}\right)^{1 / N}$. Recall that, for a set of real positive values, the geometric mean is always upper bounded by the arithmetic mean. Hence, when the total received SNR over all receive antennas, defined as $\sum_{n=1}^{N} \gamma_{n}$, is fixed, the semi-identical channel will degrade the BEP performance compared with the identical channel. Next we consider a time-varying channel with $\rho_{n}<1$ for all $n$. By letting $\gamma_{n} \gg 1$, the right-hand side of the inequality (25) approaches a constant:

$$
\begin{aligned}
F\left(\alpha \mid \theta_{p}\right) & \rightarrow \prod_{n=1}^{N}\left[1+\frac{\rho_{n}^{2} \cos ^{2}\left(\alpha+\theta_{p}\right)}{\left(1-\rho_{n}^{2}\right) P}\right]^{-M} \\
& \leq\left[1+\frac{\rho_{\min }^{2} \cos ^{2}\left(\alpha+\theta_{p}\right)}{\left(1-\rho_{\min }^{2}\right) P}\right]^{-M N} .
\end{aligned}
$$

Here, $\rho_{\min }=\min _{n}\left\{\rho_{n}\right\}$, and the equality holds when $\rho_{n}$ is the same for all $n$. This result indicates that the asymptotic irreducible error floor of differential OSTBC with optimal detection, despite its dependence on the different fluctuation rates, is independent of the unequal power distribution on different receive antennas. In the case where $\rho_{n}=\rho, \forall n$, 
it can be expected that the error floors of the semi-identical and identical channels are the same.

Note that the above Chernoff bound is only for the optimal detector. For the suboptimal detector, the value of $s$ that maximizes the $n$-th factor in $\prod_{n=1}^{N} c_{n}(s)$ with $w_{n}=1, \forall n$, is dependent on the index $n$. Therefore, the optimal $s$ can only be found by evaluating the derivative of $\prod_{n=1}^{N} c_{n}(s)$, which can be very difficult for large $N$ and $M$.

\section{NumericAl RESULTS AND Discussions}

We first study the behavior of the optimal weighting coefficients in optimal detection as a function of various system parameters. We then proceed with the BEP versus SNR plots using both analytical results and simulation results.

Throughout this section, we use the differential OSTBC for two transmit antennas and apply QPSK constellation. The number of receive antennas is fixed to $N=2$. The total average received SNR per bit over the two receive antennas is defined as $\gamma_{b}=\left(\gamma_{1}+\gamma_{2}\right) / b$, where $b$ is the transmission rate in bits per channel use and equal to 2 in our example. Let $\eta$ denote the fraction of the total average received signal energy from receive antenna 1 (and hence $1-\eta$ is from receive antenna 2). For instance, we have $\gamma_{1}: \gamma_{2}=10 \%: 90 \%$ when $\eta=0.1$. The fluctuation rates of the fading processes on the two receive antennas are assumed to be the same, i.e. $\rho_{1}=\rho_{2}=\rho$.

Figs. 1 and 2 show the values of the weights $w_{n}$, for $n=$ 1,2 , given in (6), at $\eta=0.1$ and 0.49 , respectively. The values of the weights are normalized with respect to the sum $w_{1}+w_{2}$. We first observe that the relative importance of each receive antenna depends not only on the SNR fraction at that antenna, but also on the total average SNR per bit. In particular, at low SNR the receive antenna contributing more received power has a higher weight, whereas at high SNR the receive antenna contributing less received power has a higher weight. It is also observed that when the block correlation coefficient $\rho$ approaches unity, the two antennas tend to be equally weighted at high SNR. This implies that the optimal and suboptimal differential detectors have similar performance at high SNR when the channel approaches static fading.

In Figs. 3 and 4, we plot the BEP results obtained from both theoretical analysis and computer simulation. Though block-wise time-varying fading is assumed in our theoretical analysis, we use the more realistic symbol-wise time-varying fading model in our simulations. The time variation is given by the Jakes model with normalized autocorrelation function $\mathcal{J}_{0}\left(2 \pi f_{d} T_{s} i\right)$, where $\mathcal{J}_{0}(\cdot)$ is the zeroth order Bessel function of the first kind, $f_{d} T_{s}$ is the normalized Doppler frequency, and $i$ is the index of symbol intervals. To determine the block correlation coefficient $\rho$ for computing the weights (6) used in the optimal detector, we take the average over two adjacent transmission blocks as $\rho=\sum_{i=1}^{2 M-1} \mathcal{J}_{0}\left(2 \pi f_{d} T_{s} i\right) /(2 M-1)$. The same $\rho$ is also adopted in the theoretical analysis. The analytical BEP for i.i.d channels, from [10], is plotted for comparison.

From these two figures, several observations can be made. First, it is seen that the analytical BEP derived assuming

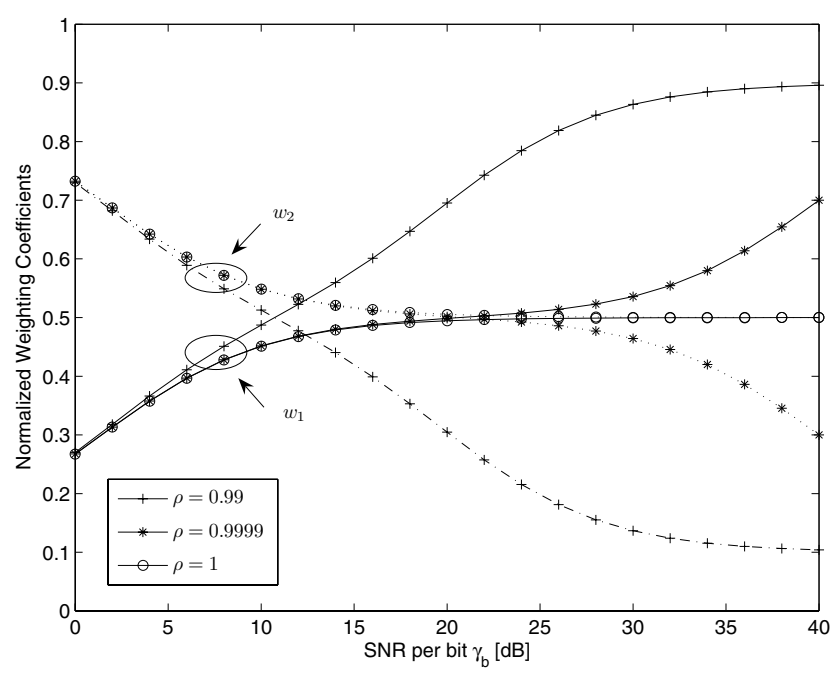

Fig. 1. Normalized optimal weighting coefficients for $N=2$ at $\eta=0.1$

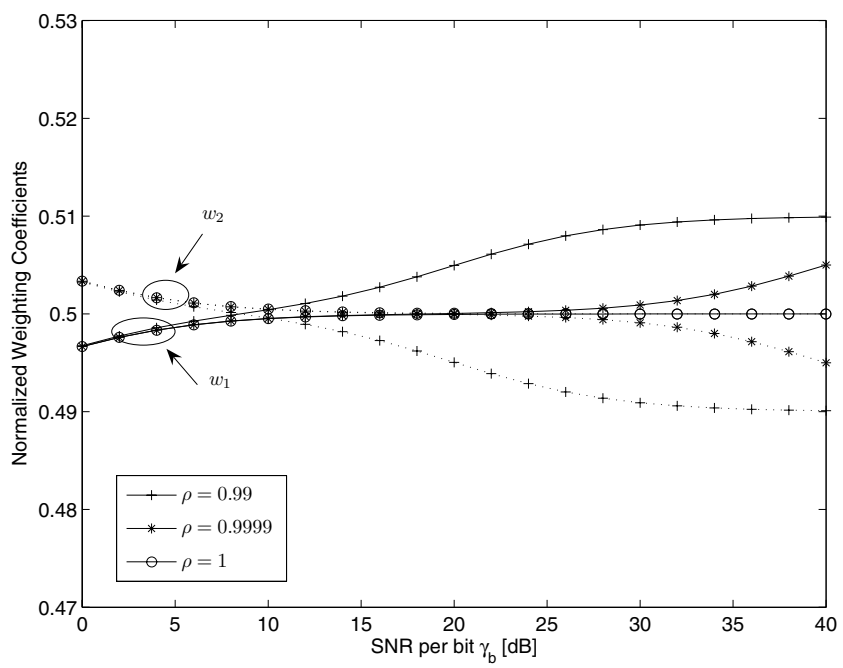

Fig. 2. Normalized optimal weighting coefficients for $N=2$ at $\eta=0.49$

block-wise time-varying fading matches very well with the simulated BEP over symbol-wise time-varying fading channels. This observation shows that the block-wise fading with block correlation coefficient determined above serves as a good model for practical time-varying channels in performance analysis. Second, the non-identical channel gain distribution among the receive antennas degrades the BEP performance. Third, the irreducible error floor of i.s.i.d channels as $\gamma_{b} \rightarrow \infty$ when the optimal detector (7) is used approaches that of the i.i.d channels. These two observations agree with the asymptotic behavior of the upper bound we obtained in Section IV. Lastly, when the normalized Doppler frequency is large $\left(f_{d} T_{s}=0.015\right)$, the optimal detector can substantially improve the BEP performance, compared with the suboptimal detector, especially in the regime of high SNR. On the other hand, when $f_{d} T_{s}$ is very small $\left(f_{d} T s=0.001\right)$, the performance of optimal and suboptimal detectors is almost identical, regardless of the unequal received powers among the receive antennas. 
This agrees with our observation from Figs. 1 and 2.

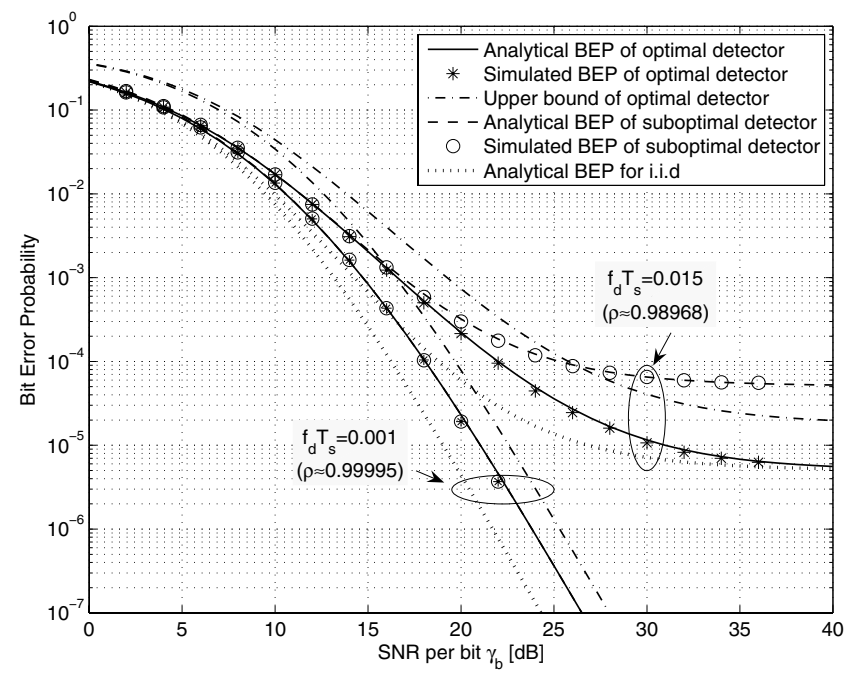

Fig. 3. Analytical and simulated BEP comparison for $M=N=2$ with QPSK at $\eta=0.1$

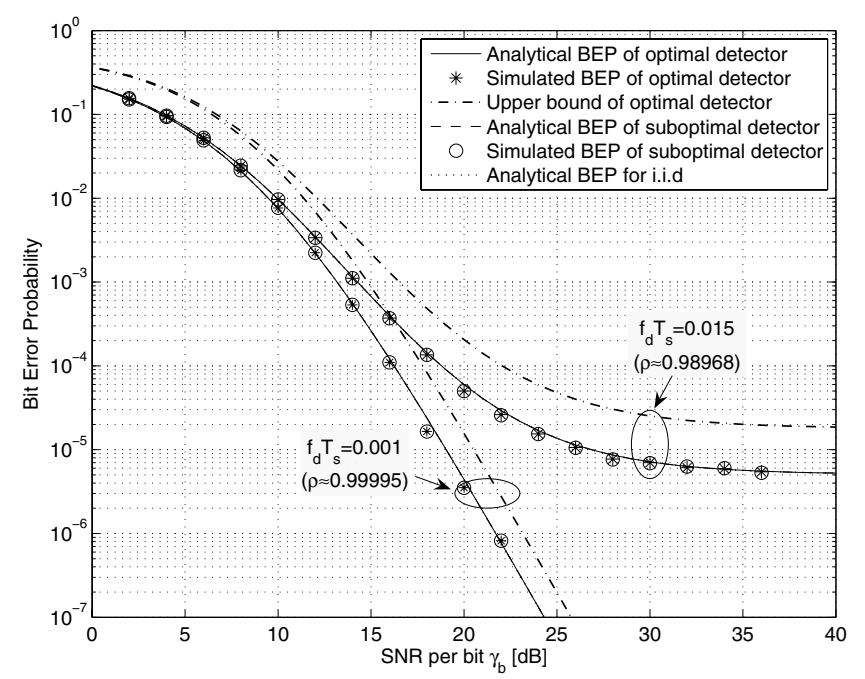

Fig. 4. Analytical and simulated BEP comparison for $M=N=2$ with QPSK at $\eta=0.49$

\section{CONCLUSION}

This paper presents the analysis of bit error probabilities for differential orthogonal space-time block codes over semiidentical MIMO Rayleigh fading channels. We take into account both unequal received SNR distribution among the receive antennas and arbitrary fluctuation rates of the blockwise time-varying channel fading processes. We show that the optimal symbol-by-symbol differential detector involves weighting the output from each receive antenna according to its channel statistical information. The exact BEP expressions for any number of transmit antennas for which OSTBCs exist are derived in the form of residues, from which the explicit and closed-form expressions for two transmit antennas are derived. Our results reveal that the non-identical channel statistics degrade the error performance compared with the identical case. We also find that the proposed optimal detector outperforms the conventional suboptimal detector when the channel fluctuates rapidly but they perform similarly in nearstatic fading channels. Though they are derived for semiidentical MIMO channels, our results can be applied to nonidentical MIMO channels under certain conditions.

\section{REFERENCES}

[1] H. Fu and P. Y. Kam, "Performance of optimum and suboptimum combining diversity reception for binary DPSK over independent, nonidentical Rayleigh fading channels," in Proc. IEEE International Conference on Comm. (ICC'05), June 2005.

[2] M. Z. Win and J. H. Winters, "Analysis of hybrid selection/maximalratio combining of diversity channels with unequal SNR in Rayleigh fading," in Proc. IEEE VTC Fall, 1999.

[3] S. M. Alamouti, "A simple transmit diversity technique for wireless communication," IEEE Journal on Selected Areas in Comm., vol. 16, pp. 1451-1458, 1998.

[4] V. Tarokh, H. Jafarkhnai, and A. R. Calderbank, "Space-time block codes from orthogonal designs," IEEE Trans. on Info. Theory, vol. 45, pp. 1456-1467, 1999.

[5] G. Ganesan and P. Stoica, "Space-time diversity using orthogonal and amicable orthogonal designs," in Prof. of International Conference on Acoustics, Speech and signal processing (ICASSP), 2000.

[6] V. Tarokh and H. Jafarkhani, "A differential detection scheme for transmit diversity," IEEE Journal on Selected Areas in Comm., vol. 18, no. 7, pp. 1169-1174, 2000.

[7] M. Tao and R. S. Cheng, "Differential space-time block codes," in Proc. IEEE Global Telecommunications Conference (GLOBECOM), 2001.

[8] G. Ganesan and P. Stoica, "Differential detection based on space-time block codes," Wireless Personal Comm., vol. 21, pp. 163-180, 2002.

[9] C. Gao and A. M. Haimovich, "BEP analysis of MPSK space-time block codes with differential detection," IEEE Comm. Letters, vol. 7, no. 7, pp. 314-316, July 2003.

[10] T. P. Soh, P. Y. Kam, and C. S. Ng, "Bit error probability for orthogonal space-time block codes with differential detection," IEEE Trans. on Comm., vol. 53, no. 11, pp. 1795-1798, Nov. 2005.

[11] E. Chiavaccini and G. M. Vitetta, "Further results on differential spacetime modulation," IEEE Trans. on Comm., vol. 51, no. 7, pp. 1093-1101, 2003.

[12] G. Ganesan and P. Stoica, "Space-time block codes: a maximum SNR approach," IEEE Trans. on Info. Theory, vol. 47, no. 4, pp. 1650-1656, May 2001.

[13] A. V. Geramita and J. Seberry, Orthogonal designs, quadratic forms and Hadamard Matrices. Marcel Dekker: New York and Basel, 1978, vol. 43 of Lecture Notes in Pure and Applied Mathematics.

[14] Z. Chen, G. Zhu, J. Shen and Y. Liu, "Differential space-time block codes from amicable orthogonal designs," in Proc. IEEE Wireless Comm. and Networking, March 2003.

[15] R. A. Horn and C. R. Johnson, Matrix analysis. New York : Cambridge University Press, 1985.

[16] P. Y. Kam, "Bit error probabilities of MDPSK over the nonselective Rayleigh fading channel with diversity reception," IEEE Trans. on Comm., vol. 39, no. 2, 1991.

[17] M. Brehler and M. K. Varanasi, "Asymptotic error probability analysis of quadratic receivers in Rayleigh-fading channels with applications to a unified analysis of coherent and noncoherent space-time receivers," IEEE Trans. on. Info. Theory, vol. 47, no. 6, pp. 2383-2399, Sept 2001.

[18] G. M. Vitetta, U. Mengali, and D. P. Taylor, "Optimal noncoherent detection FSK signals transmitted over linearly time-selective Rayleigh fading channels," IEEE Trans. on Comm., vol. 45, no. 11, pp. 14171426, Nov. 1997

[19] J. G. Proakis, Digital Communications, 4th ed. New York: McGrawHill, 2001. 